\title{
Plan de Acción Tutorial para Alumno de Planes de Estudios a Extinguir en la Escuela Técnica Superior de Ingenierías Informática y de Telecomunicación (TIPAT)
}

\author{
M. Rodríguez Álvarez ${ }^{1}$; M. I. García Arenas ${ }^{1}$; P. Paderewski Rodríguez, ${ }^{2}$; E. M. Ra- \\ mos Ábalos ${ }^{3}$; J. García Miranda, ${ }^{4}$; M. A. Rubio Escudero ${ }^{5}$; N. Rico Castro ${ }^{3}$; P. A. \\ Castillo Valdivieso ${ }^{1}$.
${ }^{I}$ Departamento de Arquitectura y Tecnología de Computadores. E.T.S. Ingenierías Informática y de Telecomunicación. Universidad de Granada.
${ }^{2}$ Departamento de Lenguajes y Sistemas Informáticos. E.T.S. Ingenierías Informática y de Telecomunicación. Universidad de Granada.
${ }^{3}$ Departamento de Estadística e Investigación Operativa. Facultad de Ciencias. Universidad de Granada
${ }^{4}$ Departamento de Álgebra. E.T.S. Ingenierías Informática y de Telecomunicación. Universidad de Granada.
${ }^{5}$ Departamento de Ciencias de la Computación e Inteligencia Artificial. E.T.S. Ingenierías Informática y de Telecomunicación. Universidad de Granada. \\ mgarenas@ugr.es, patricia@ugr.es, ramosa@ugr.es, jesusgm@ugr.es, mrubio@ decsai.ugr.es, \\ nrico@ugr.es, pedro@ atc.ugr.es, $\underline{\text { manolo@ugr.es }}$
}

Resumen. En este trabajo se presenta un Plan de Acción Tutorial (TIPAT) para alumnos de planes de estudio a extinguir en la Escuela Técnica Superior de Ingenierías Informática y de Telecomunicación (ETSIIT) de la Universidad de Granada. TIPAT se ha venido desarrollando a lo largo de tres cursos académicos. Tras una introducción se presenta el Plan de Acción Tutorial, el desarrollo del trabajo, así como los resultados obtenidos y el beneficio que ha supuesto para los estudiantes la realización de este proyecto.

Palabras clave: Plan de acción tutorial (PAT), tutoría, orientación.

\begin{abstract}
This work shows a Tutorial Plan (named TIPAT) for students in extinct studies in the Faculty of Computer Science and Telecommunication Engineering of the University of Granada. TIPAT has been developed over three academic years. After an introduction this work presents the Tutorial Plan, development work, as well as the results and the benefits it has brought to students carrying out this project.
\end{abstract}

Keywords: Tutorial Plan, tutoring, mentoring. 


\section{Antecedentes}

TIPAT nace con el objetivo de prestar ayuda a los estudiantes de planes de estudio a extinguir de la Escuela Técnica Superior de Ingenierías Informática y de Telecomunicación (ETSIIT) de la Universidad de Granada que tienen pendientes asignaturas de las que no existen ya docencia presencial. Debido a las peculiares necesidades de estos estudiantes, se puso en marcha en el curso 2010-2011 este Plan de Acción Tutorial, conocido como TIPAT que ha continuado durante los cursos académicos 20112012 y 2012-2013. La propuesta original se centra en la acción tutorial hacia estudiantes que se encuentran matriculados de asignaturas que se han extinguido, dado que están cursando un plan de estudios que está siendo sustituido. Este problema tiene una duración temporal limitada; comienza en el curso 2010-2011 y se prolonga, al menos, durante los cuatro años en que progresivamente van suprimiéndose los cursos del plan antiguo.

En el curso 2012-2013 dejarán de impartirse las asignaturas de tercer curso de las tres titulaciones asociadas a los estudios de Informática y de la de los estudios de Ingeniero de Telecomunicación. Previamente, durante los cursos 2010-2011 y 20112012 dejaron de impartirse las asignaturas de primer y segundo curso. Esto hace que en las titulaciones de Ingeniero Técnico en Informática de Sistemas e Ingeniero Técnico en Informática de Gestión ya se haya extinguido totalmente el plan de estudios sin que se imparta docencia presencial. En las titulaciones de ciclo largo, a saber Ingeniero en Informática e Ingeniero de Telecomunicación todavía persiste la docencia presencial en los cursos $4^{\circ}$ y $5^{\circ}$. La desaparición de la docencia presencial de estas asignaturas conlleva una pérdida general en el sistema de apoyo y ayuda que recibe el estudiante en comparación con la orientación habitual que se establece durante las clases presenciales.

El Proyecto TIPAT que se puso en marcha durante el curso 2010-2011 presenta, mediante distintas vías de actuación, una red de apoyo, información y orientación para los alumnos que se encuentran matriculados de asignaturas en cursos sin docencia. La continuación de este proyecto tiene por objeto ofrecer esta ayuda a los estudiantes que durante el curso 2012-2013 se van a encontrar sin docencia de asignaturas de tercer curso, y, posiblemente, también de primero y segundo.

Algunas cifras a tener en cuenta sobre las asignaturas que a extinguir en segundo curso son:

- Número de asignaturas que se extinguieron en el curso 2010-2011: 42

- Número de matrículas realizadas en asignaturas sin docencia en el curso 20102011: 2177

- Número de asignaturas que se extinguieron en el curso 2011-2012: 37

- Número de matrículas realizadas en asignaturas sin docencia en el curso 20112012: 3136

- Número de asignaturas sin docencia en el curso 2012-2013: 133 
Tabla 1. Asignaturas a extinguir. Curso 2012-2013.

\begin{tabular}{|l|c|c|c|}
\hline \multicolumn{2}{|l|}{ Asignaturas a extinguir en 2012-2013 } & \multicolumn{1}{l|}{} \\
\hline Titulación & $\begin{array}{c}\text { Primer } \\
\text { cuatrimestre }\end{array}$ & $\begin{array}{c}\text { Segundo } \\
\text { cuatrimestre }\end{array}$ & Total \\
\hline Ingeniero en Informática & 14 & 14 & $\mathbf{2 8}$ \\
\hline I.T. Informática de Sistemas & 21 & 19 & $\mathbf{4 0}$ \\
\hline I.T. Informática de Gestión & 21 & 19 & $\mathbf{4 0}$ \\
\hline Ingeniero Telecomunicación & 13 & 12 & $\mathbf{2 5}$ \\
\hline
\end{tabular}

El hecho de que desaparezca tan elevado número de asignaturas es debido a la gran oferta de asignaturas optativas que hay en tercer curso de las cuatro titulaciones.

Los Departamentos que tienen asignadas las asignaturas que desaparecen son:

- Álgebra

- Análisis Matemático.

- Arquitectura y Tecnología de Computadores.

- Ciencias de la Computación e Inteligencia Artificial.

- Economía Financiera y Contabilidad

- Electromagnetismo y Física de la Materia Condensada.

- Electrónica y Tecnología de Computadores.

- Estadística e Investigación Operativa.

- Física Aplicada.

- Lenguajes y Sistemas Informáticos.

- Matemática Aplicada.

- Organización de Empresas

- Teoría de la Señal, Telemática y Comunicaciones.

Dados los datos de los cursos 2010-2011 y 2011-2012, se puede estimar que para el curso 2012-2013 habrá más de 2500 matrículas en asignaturas sin docencia. El número de estudiantes que se ven afectados por esta situación puede ser muy elevado, más de 400 estudiantes. Por otra parte, en la puesta en marcha de TIPAT, durante los cursos 2010-2011 y 2011-2012 se recibieron más de 400 solicitudes de participación en cada curso, lo que sugiere que una gran parte de los estudiantes que se van a encontrar sin docencia presencial de tercer curso también demandarán la ayuda de TIPAT.

\section{Descripción}

En este Plan de Acción Tutorial, TIPAT, colaboran estudiantes, profesores y personal de administración y servicios vinculados con la Escuela Técnica Superior de Ingenierías Informática y de Telecomunicación de la Universidad de Granada. Se enmarca 
dentro de los Proyectos de Innovación Docente que financia el Vicerrectorado de Ordenación Académica y Profesorado de la Universidad de Granada a través del Secretariado de Innovación Docente.

En la primera etapa de TIPAT, desarrollada durante el curso 2010-2011, formaron parte del proyecto un total de 345 estudiantes, 42 profesores y 2 trabajadores del área de Administración; en total 389 participantes vinculados con la Escuela Técnica de Ingenierías Informática y de Telecomunicación.

En su segunda etapa, desarrollada durante el curso 2011-2012, el número de participantes es de 203 (130 estudiantes, 71 profesores y 2 trabajadores del área de Administración). Además, en cada uno de los dos cursos académicos en los que TIPAT ha funcionado, ha contado con la ayuda técnica de un estudiante en prácticas, a través del Centro de Promoción de Empleo y Prácticas, del Vicerrectorado de Estudiantes de la Universidad de Granada.

\section{Objetivos}

Los objetivos de cualquier proyecto de estas características deben ser claros y no deben ser muchos para evitar abarcar más tareas de las que realmente se van a llevar a cabo. Además teniendo en cuenta que se trata de un proyecto de orientación y tutoría el objetivo general es claro: guiar a los alumnos en el plano personal, académico, social y profesional como una parte más de su proceso formativo. Teniendo en mente este primer objetivo general, se puede detallar y afinar más adecuando este objetivo general al ámbito de este proyecto. Y en este ámbito particular el objetivo final será orientar y colaborar con el alumnado en la preparación del temario de las distintas asignaturas sin docencia o a extinguir dentro del plan de estudios al que pertenezcan dichas asignaturas, persiguiendo el superar los exámenes de cada una de las asignaturas pendientes.

Detallando este objetivo general aún más, se pueden extraer una serie de objetivos específicos:

- Evaluar la situación de partida del alumno, para determinar sus conocimientos, debilidades, necesidades y potencialidades.

- Facilitar técnicas de estudio y de reciclaje de conocimientos.

- Informar de la actualización de los contenidos del temario así como de cambios en la forma, tipo o puntuación del examen.

- Crear un espacio de encuentro, en el que se transmitan los problemas y dificultades a los que se enfrentan los alumnos, y en el que se estimulen habilidades y actitudes positivas.

- Apoyar al alumno y realizar una labor de nexo de unión y vía de comunicación con las asignaturas extinguidas del plan antiguo.

- Potenciar el alumno activo y diseñar con él un itinerario de preparación de asignaturas. 


\section{Actividades Realizadas}

Dentro del desarrollo de este proyecto se planificaron varias actividades a llevar a cabo a lo largo del curso académico, tanto para los estudiantes participantes como para los profesores tutores. En el caso de los profesores tutores, se impartió una charla inicial en la que se informaba del modo de actuación que se iba a seguir en el caso de que los alumnos que no atendieran a los tutores o en el caso de tutores que no atendieran debidamente a los alumnos. Además en esta charla se introdujeron las novedades con respecto a las actividades planificadas para los alumnos para que desde los primeros días de desarrollo del proyecto los profesores tutores tuvieran conocimiento de las actividades propuestas.

En el caso de los alumnos, se planificaron tres actividades a lo largo del todo el curso. La primera de ellas era una sesión informativa a nivel administrativo del procedimiento que se seguía para las asignaturas sin docencia, aclarando temas administrativos referentes al número de convocatorias disponibles por curso académico, o a lo largo de todos los años en los que el estudiante se hubiera matriculado de ella. Además se informó de la normativa vigente para exámenes, precios de matriculación, matriculas de asignaturas que constan como optativas en el plan de estudio a extinguir. La sesión fue impartida por el personal que colabora con el proyecto y que a la vez forma parte de la secretaria de la Escuela Superior de Ingeniería Informática y por lo tanto conoce a fondo los problemas que los estudiantes iban planteando. Esta sesión se desarrollo a modo de seminario y fue totalmente voluntario para los alumnos que quisieron asistir.

Por último, se organizaron dos sesiones informativas sobre las asignaturas que durante el curso académico 2011-2012 estaban sin docencia por primera vez. La primera de ellas se impartió en el primer cuatrimestre incluyendo las asignaturas cuya evaluación estaba prevista a final del primer cuatrimestre y la segunda para las asignaturas del segundo cuatrimestre. En ambos casos, sólo se impartieron charlas para las asignaturas que tenían un mínimo de personas matriculadas y cuyo profesor responsable de la evaluación estaba dispuesto a impartirla. Hay que destacar, que en todos los casos los profesores realizaron estas actividades de forma totalmente voluntaria y que en ningún caso hubo profesores que se negaran a hacerla después de que el grupo coordinador del proyecto se lo pidiera.

\section{Resultados, Productos y Beneficios Generados por el Proyecto}

La valoración de los resultados del proyecto se ha realizado a partir de un cuestionario de valoración por parte de los estudiantes, la creación de un buzón de sugerencias, la apertura de un foro de debate en la plataforma web y reuniones del equipo de coordinación.

\subsection{Resultados de la evaluación del aprendizaje de los estudiantes}

De los resultados de la encuesta se deduce como las sesiones de orientación, las actividades organizadas (charlas de presentación de asignaturas, talleres y sesiones de 
repaso) y la utilización de la página web del proyecto (ver figura 1) han sido muy beneficiosas para el estudiante.

\subsection{Resultados de la evaluación interna e instrumentos utilizados.}

Los resultados de la encuesta han sido muy positivos, destacando que un $77.8 \%$ del alumnado encuestado considera muy importante la orientación universitaria y un 83.3\% que es muy importante la existencia de un plan de acción tutorial como TIPAT.

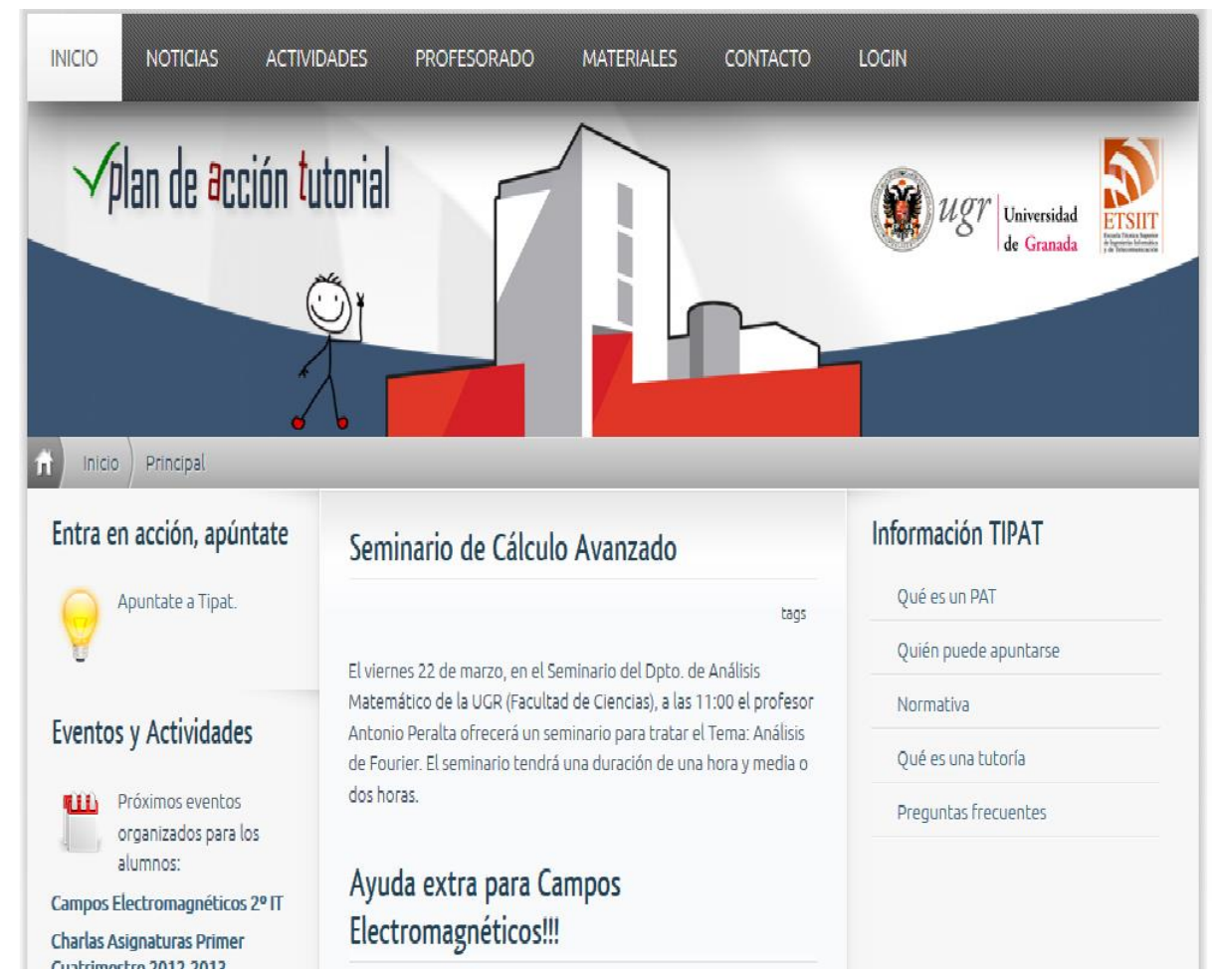

Fig. 1. Sitio web del plan de acción tutorial 


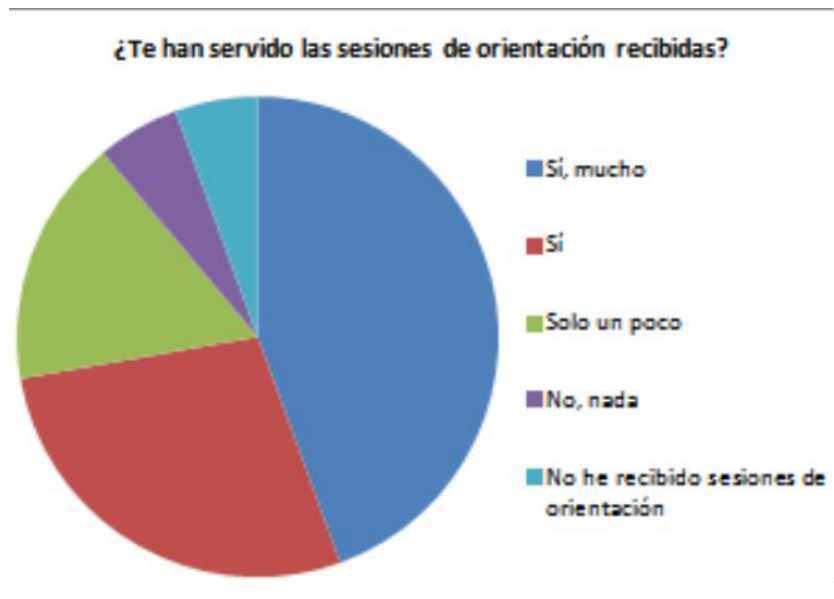

¿Cuál es tu valoración sobre las charlas de presentación de las asignaturas?

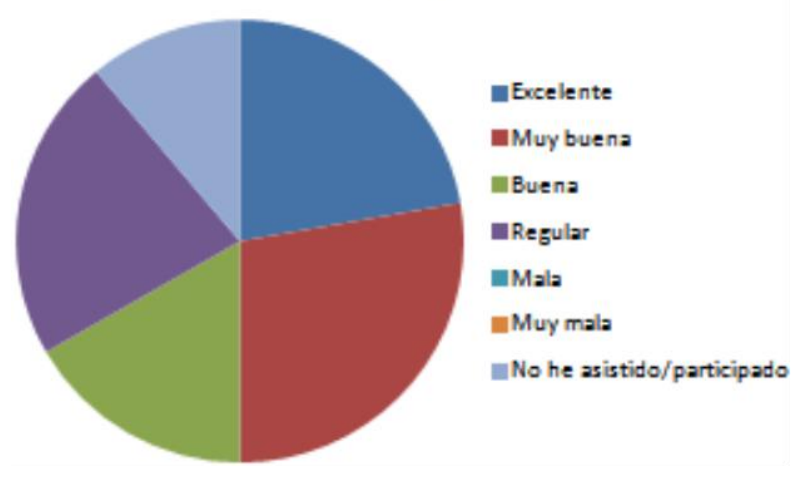

¿Cuál es tu valoración sobre las sesiones de repaso y dudas que se han organizado?

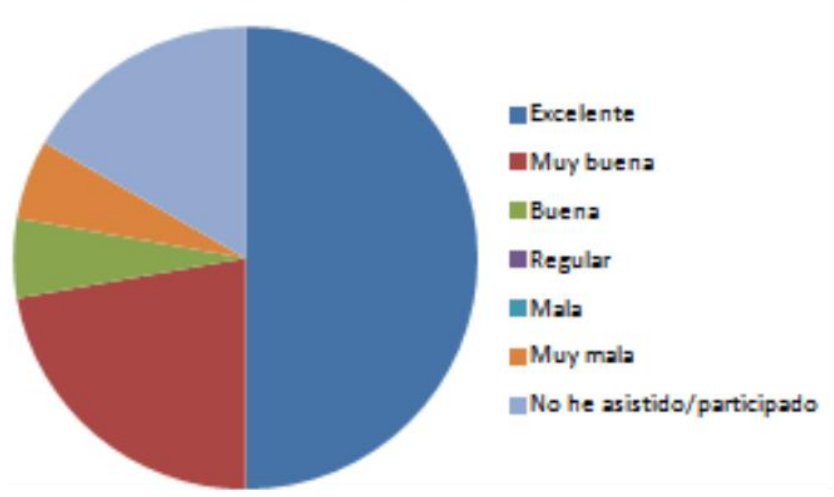

Fig. 2. Resultados de la evaluación del aprendizaje de los estudiantes 


\subsection{Resultados de la evaluación externa e instrumentos utilizados}

Dado que la valoración general fue muy buena en todas las ocasiones en las que se presentó la primera edición de TIPAT en congresos, reuniones y seminarios a nivel nacional e internacional ([1], [2], [3] y [4]), se ha seguido la misma dinámica con la segunda edición de TIPAT, volviéndose a obtener un opinión muy positiva en el foro internacional presentado ([5]).

Para conocer la opinión del alumnado se ha creado en la plataforma virtual un buzón de sugerencias individualizado y privado, de forma que el alumno pueda expresar sus quejas, necesidades, impresiones y sugerencias en cualquier momento.

Además como modalidad complementaria al buzón de sugerencias, se ha abierto un foro de debate que permitirá visibilidad pública por parte de alumnos y profesores de los comentarios que se manden a la plataforma. De esta manera, las sugerencias planteadas serán moderadas entre todos, y el alumno tendrá una mejor percepción de su participación en la gestión de la experiencia.

\section{Valoración Global}

En general la puesta en marcha y el desarrollo del proyecto ha sido muy positiva, consiguiéndose todos los objetivos propuestos, y en particular para los alumnos tal y como muestran los resultados de la encuesta presentada en la figura 2.

\section{Bibliografía}

1. García Arenas, M.I.; Paderewski Rodríguez, P.; Ramos Ábalos, E.M.; García Miranda, J.; Rubio Escudero, M.A.; Rico Castro, N.; Castillo Valdivieso, P.A.; Rodríguez Álvarez, M.: "Implantación de un Plan de Acción Tutorial para alumnos de planes de estudio a extinguir de la ETSIIT de la Universidad de Granada." II Jornadas sobre Innovación Docente y adaptación al Espacio Europeo de Educación Superior en las Titulaciones Técnicas. Universidad de Granada. Granada, 26 y 29 de Septiembre de 2011. ISBN 978-84-15418-00-9, pp. 419-422.

2. Paderewski Rodríguez, P.; García Miranda, J.; Rubio Escudero, M.A.; Cruz Corona, C.; Montes Soldado, R.A.; Rodríguez Álvarez, M.: "Plan de Acción Tutorial en la Universidad de Granada. Primeros resultados." II Congreso Internacional de Docencia Universitaria (CIDU 2011). Vigo (España), 30 de Junio a 2 de Julio de 2011.

3. Rico Castro, N.; Castillo Valdivieso, P.A.; Rubio Escudero, M.A.; Paderewski Rodríguez, P.; García Arenas, M.I.; Ramos Ábalos, E.M.; García Miranda, J.; Rodríguez Álvarez, M.: "A new tutorial system for computer science and telecommunication engineering students" Congreso New Perspectives in Science Education. Florencia (Italia), 8 y 9 de Marzo de 2012.

4. Rico Castro, N.; García Arenas, M.I.; Ramos Ábalos, E.M.; Rubio Escudero, M.A.; Romero García, S.F.; Rodríguez Álvarez, M.: "Plan de acción tutorial para alumnos de planes de estudio a extinguir en la Escuela Técnica Superior de Ingenierías Informática y de Telecomunicación." Actas de las I Jornadas sobre Inno- 
vación Docente y Adaptación al EEES en las Titulaciones Técnicas. Granada, 9 y 10 de Septiembre de 2010, pp. 215 a 218.

5. Díez Sánchez, M.A.; Rico Castro, N.; García Arenas, M.I.; Paderewski Rodríguez, P.; Castillo Valdivieso, P.A.; Rodríguez Álvarez, M.: "Sistema web de apoyo para el desarrollo de un Plan de Acción Tutorial.” III Jornadas sobre Innovación Docente y adaptación al Espacio Europeo de Educación Superior en las Titulaciones Técnicas. Universidad de Granada. Granada, 20 y 21 de Septiembre de 2012. ISBN 978-84-15418-73-3, pp. 203-207. 